\title{
The Use of Preseptal Transconjunctival Approach in the Surgical Management of the Infraorbital Rim and Floor
}

\section{Fractures Technical Note}

\section{Adil E*, Safaa T, Asmae B, Ayoubi Fahd EI and Mohamed Rachid G \\ Department of ENT and Maxillo-facial, Mohamed VI University Hospital, Morocco}

*Corresponding author: Eabdenbitsen Adil, Department of ENT and Maxillo-facial, Mohamed VI University Hospital, Morocco, Tel: 00212661255075; Email: dr.ea_adil@yahoo.fr

\section{Summary}

The transconjunctival approach is used for the exploration and the treatment of the orbital floor fractures. There are two main surgical routes to this approach: retroseptal and preseptal dissection. The retroseptal approach offers a greater exposure of the orbital floor and the infraorbital rim, however, compared to the preseptal approach, it is associated with higher rate of lower eyelid complications due to the disturbance of the inferior conjunctivae's connective tissue. The objective of this study is to evaluate the efficacy of the preseptal route in the transconjunctival approach.

\section{Introduction}

The surgical access to the Infraorbital rim is generally accomplished by the transcutaneous approach that offers the surgeon a good enough intraoperative visibility to make an anatomical reduction and to fix the fractured bone with miniplate osteosynthesis, however, the skin incision involves the risk of scarring problems as well as other complications such as ectropion majored by the subciliary approach. The preseptal transconjunctival approach can be performed either independently or combined with an additional transcaruncular approach, or with lateral canthotomy/cantholysis.

This approach wasn't initially described until the 1971 when Tenzel and Miller used this incision for the repair of the orbital floor slight fractures [1]. In 1974, CONVERSE made the transconjunctival incision gain a wide acceptance as an approach for the surgical management of orbital floor fractures; furthermore, he described the two different routes to this approach: retroseptal and preseptal incisions.

The versatility of these conjunctival approaches contributed in promoting their use for a surgical access to the orbital skeleton in order to treat different pathological conditions, such as orbital fractures, orbital tumors and infections, orbital decompression in certain cases of ophthalmoplegia, as well as in the secondary orbital reconstructions. The conjunctival approaches are also used in the rejuvenation surgery (lower eyelid blepharoplasty).

The use of conjunctival approach in severe orbital fractures remains controversial, because it may not provide enough exposure. Nevertheless, a literature review suggests that this limitation of exposure in the conjunctival approach is more likely attributed to the lack of the surgeon's experience rather than real limitation of the approach itself [2]. The purpose of this is to study is to highlight, through the experience of our department, the advantages of the preseptal transconjunctival approach in the treatment of these fractures comparing it with the transcutaneous approaches, and also to give a step by step description of it surgical technique.

\section{Surgical technique}

A forced diction test is first performed by grasping the conjunctiva near the limbus with a fine Adson forceps to 
evaluate the restriction of ocular motility caused by muscular incarceration in the site of fracture (Figure 1).

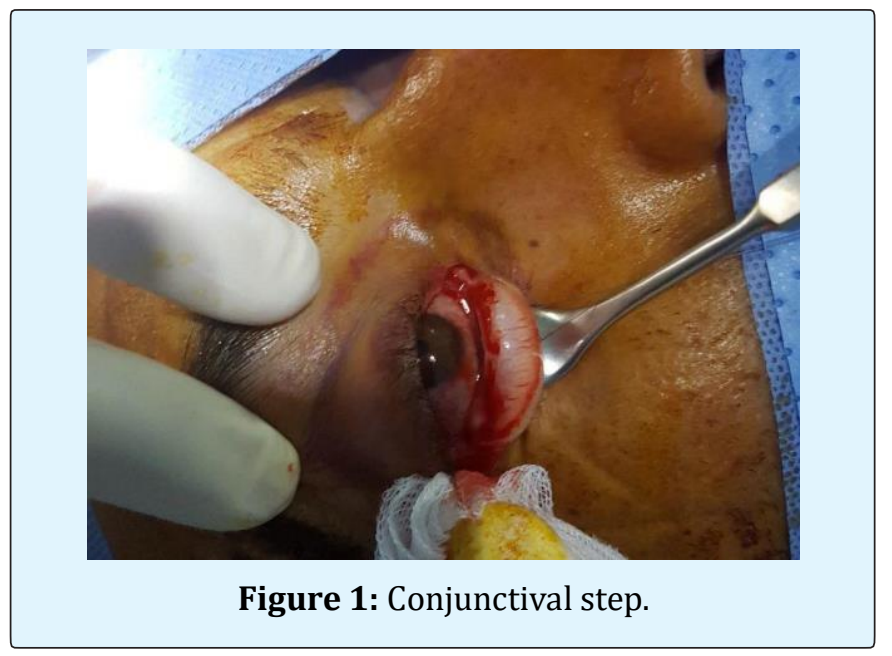

After the lower eyelid is retracted with a Joseph double hook retractor or palpebral retractor, the incision is performed with a scalpel blade, 2 to $3 \mathrm{~mm}$ underneath the lower border of the tarsal plate from the carbuncular region to the lateral central region. Hemostasis of the subconjunctival layer is achieved with bipolar electro cautery (Figure 2).

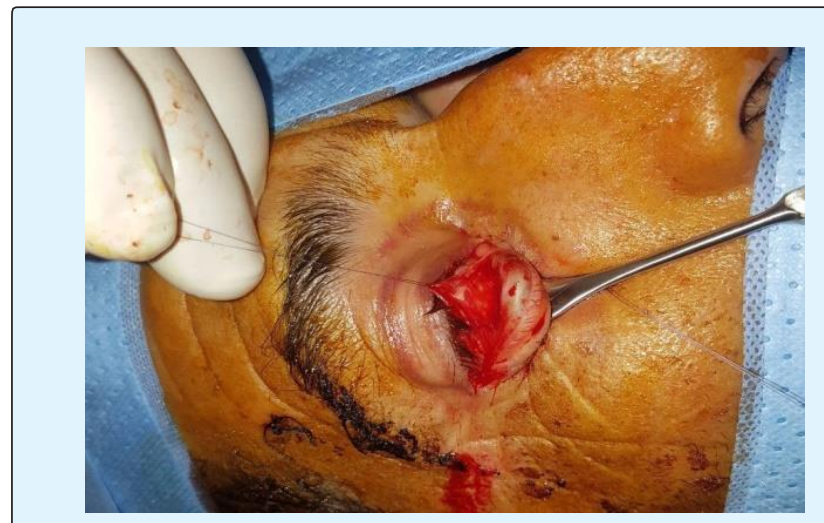

Figure 2: Subconjunctival step.

A subconjunctival dissection is performed with Steven's scissor. The preseptal cranial conjunctival flap is identified and separated from the caudal flap that's covered by the orbicular muscle's facia. An avascular plane between these two flaps is opened with the scissors toward the inferior orbital rim. The preseptal conjunctival flap and the globe are retracted by a malleable retractor, while the caudal conjunctival flap and the lower eyelid are pulled with a palpebral retractor. And so the inferior orbital rim is totally exposed.

\section{Subperiostal step (Figure 3)}

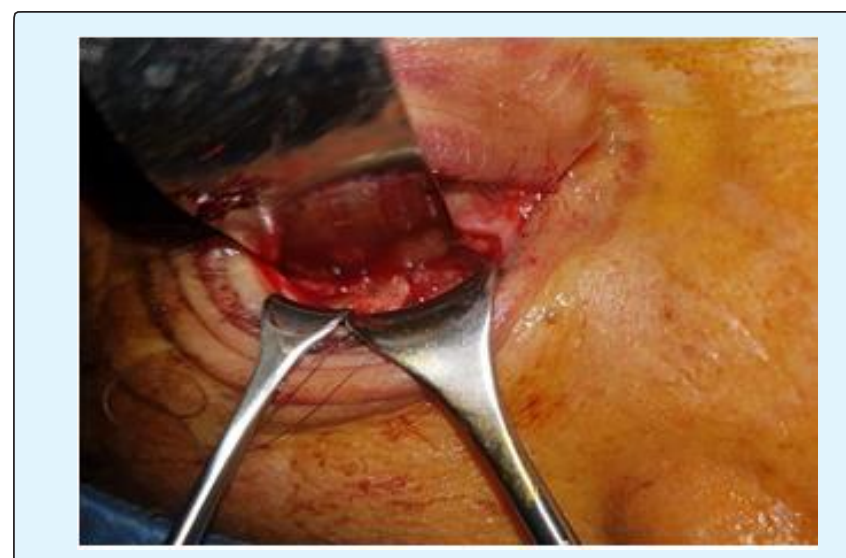

Figure 3: The periosteal incision is performed over the inferior orbital rim; the periosteum is elevated with a periosteal elevator toward the orbital floor.

The incarcerated per orbital tissues in the fracture site are repositioned, reduction of the bone fracture and osteosynthesis with titanium miniplates (Figure 4).

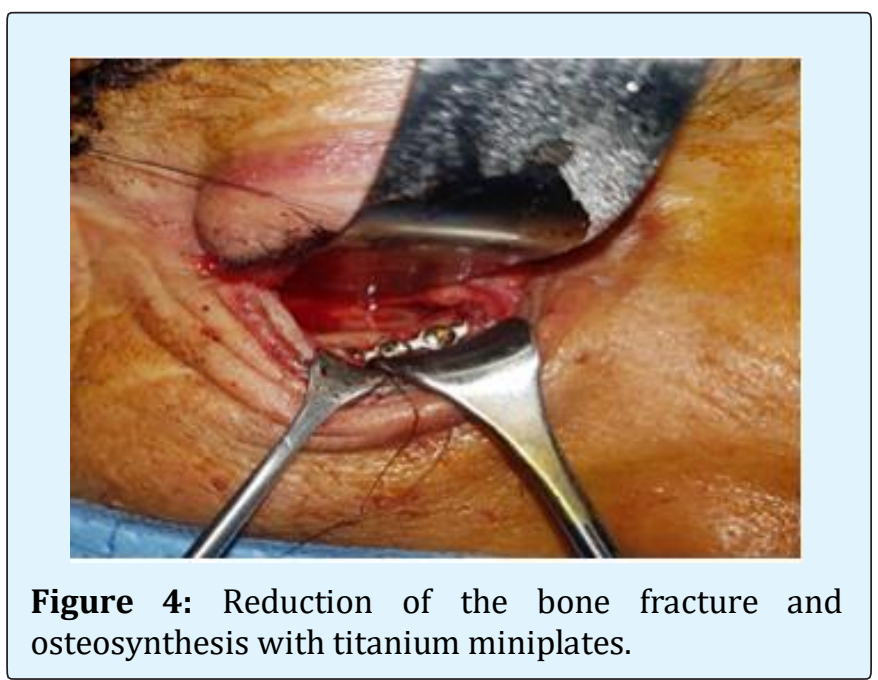

\section{Wound closure step}

The periorbita is sutured to the periosteum over the inferior orbital rim using interrupted 5-0 vicryl sutures. The conjunctiva is closed with a running 6-0 fast resorb able Maxon suture. 


\section{International Journal of Surgery \& Surgical Techniques}

\section{Discussion}

The transcutaneous approaches were massively used for the treatment of infraorbital rim and the orbital floor fractures. The subciliary approach, first described by converse in 1944, was the most commonly used given that it offers a quick and comfortable access to the infraorbital rim and the orbital floor. However the subciliary approach is linked to a high risk of complications, ectropion being the most frequent one [3]. On the other hand, the sub tarsal approach described by converse in 1960, is considered to be the safest cutaneous approach, seeing that the risk of ectropion is lower, although hypertrophic scar may occur during the healing process.

The transconjunctival approaches have tremendously gained in popularity during these last two decades given the excellent surgical exposure of the orbital floor while avoiding post-operative complications compared to transcutaneous approaches. The need of a better knowledge of the orbital anatomy is however necessary. The transconjunctival approach can be proceeded either by preseptal or retroseptal incision. The retroseptal approach provides as a main advantage the rapid access to the infraorbital rim by passing through the orbital fat, however, the orbital fat can be annoying during the surgical procedure, which represents, besides the risk of ocular motility disorder and exophthalmos, a major disadvantage $[4,2]$.

The preseptal approach is considered to be more anatomical, enabling to preserve the integrity of the tissues especially the orbital septum. It is important to remember that the conjunctival incision must be placed at $3 \mathrm{~mm}$ beneath the tarsal plate to avoid a vertical shortening of this plate and eventually the development of fibrosis. According to the literature, the transconjunctival approach is related to complications in only $2.1 \%$ of cases, with ectropion as the main complication, and most rarely lachrymal ducts injury and conjunctival pyogenic granuloma $[5,6]$.
The preseptal incision can also be combined with a transcaruncular approach and lateral canthotomy, to permit a circumferential exposure of the orbital surface [2]. In conclusion, the preseptal approach represents the current trend for being a more anatomical route that permits an easily access to the orbital floor and the infraorbital rim through an avascular plane, along with decreased chances of lower eyelid complications.

\section{References}

1. Tenzel RR, Miller GR (1971) Orbital blow-out fracture repair, conjunctival approach. Am J Ophthalmol 71(5): 1141-1142.

2. Kushner GM (2006) Surgical approaches to the infraorbital rim and orbital floor: the case for the transconjunctival approach. J Oral Maxillofac Surg 64(1): 108-110.

3. Ridgway EB, Chen C, Colakoglu S, Gautam S, Lee BT (2009) The incidence of lower eyelid malposition after facial fracture repair: a retrospective study and meta-analysis comparing subtarsal, subciliary, and transconjunctival incisions. Plast Reconstr Surg 124(5): 1578-1586.

4. Converse JM, Firmin F, Wood-Smith D, Friedland JA (1973) The con- junctival approach in orbital fractures. Plast Reconstr Surg 52(6): 656-657.

5. Mullins JB, Holds JB, Branham GH, Thomas JR (1997) Complications of the transconjunctival approach. A review of 400 cases. Arch Otolaryngol Head Neck Surg 123(4): 385-388.

6. Tessier P (1973) The conjunctival approach to the orbital floor and maxilla in congenital malformation and trauma. J Maxillofac Surg 1(1): 3-8. 